\title{
ARTICLE
}

\section{An application of genetic multi-objective optimization algorithm to neutron spectrum unfolding problem}

\author{
Myeong Hyeon Woo ${ }^{\mathrm{a}}$, Jae Hyun Kimª, Jong Woo Kim ${ }^{\mathrm{b}}$, Che Wook Yimª Jae Yong Lee ${ }^{\mathrm{a}}$, Do Hyun Kimª \\ Quang Huy Khuat ${ }^{\mathrm{a}}$, Bo Kyun $\mathrm{Seo}^{\mathrm{c}}$, Chang Ho Shin ${ }^{\mathrm{a}}$ and Jong Kyung Kim ${ }^{\mathrm{a}^{*}}$ \\ ${ }^{a}$ Department of Nuclear Engineering, Hanyang University, 222 Wangsimni-ro, Seongdong-gu, Seoul, Republic of Korea; ${ }^{b}$ Korea \\ Atomic Energy Research Institute (KAERI), 111 Daedeok-daero 989 Beon-Gil, Yuseong-gu, Daejeon, Republic of Korea; ${ }^{c}$ Korea \\ Institute of Nuclear Safety, 62 Gwahak-ro, Yuseong-gu, Daejeon, Republic of Korea
}

\begin{abstract}
Since it is difficult to directly measure neutron energy, the neutron spectrum is estimated from the specific responses of the neutron reaction. Although several algorithms have been proposed to reconstruct the neutron spectrum, no attempt has been made to apply a multi objective optimization technique. This study is based on the idea that reconstructing the spectrum by taking into consideration various prior information simultaneously enables to obtain more reasonable results. The genetic multi-objective optimization scheme was applied to derive the Pareto front of spectrum from activation foil responses. The two objectives of maximizing the Shannon information entropy of the neutron spectrum and minimizing the relative error of the responses were considered. By applying the algorithm, we were able to successfully reduce the solution candidates to Pareto front and improve the validity of the unfolded spectrum.
\end{abstract}

Keywords: spectrum unfolding; genetic algorithm; multi object optimization; activation foil; Pareto optimal

\section{Introduction}

Neutron spectrum is usually reconstructed from the consequence of neutron reactions, and this process is called neutron spectrum unfolding. Since the neutron spectrum is important for radiation safety and measurement calibration, the spectrum has been reconstructed by using Boner spheres, activation foils or others $[1,2]$. These various responses are used for unfolding, but the mathematical principle is the same.

Spectrum unfolding is the finding of the input from the response, which is mathematically in the category of the inverse problem [3]. It is often difficult to find the solution because there can be multiple inputs giving the same results. Therefore, in order to derive reasonable candidates from a large number of inputs, the solution should contain prior information such as physical constraints, statistical evaluation, and initial guess spectrum.

There are various ways of unfolding depending on how the information is considered. For example, there are several as follows: Regularization method to avoid overfitting by limiting variables; Methods of reflecting statistical information such as Shannon Entropy and Fisher information into the spectrum; Bayesian inversion that reflects initial guess spectrum using Bayesian statistics [4-8].

Although each of these methods reflected important information, there was no attempt to reflect multiple information at the same time. It is expected that reconstructing the spectrum considering multiple constraints will be more reasonable. In this study, the solution on the Pareto front, a set of solutions maximizing multiple objectives, was derived by applying a genetic algorithm to the unfolding problem and its effectiveness was evaluated.

\section{Spectrum unfolding algorithm}

\subsection{Mathematical representation of spectra unfolding}

The response induced by the neutron flux can be expressed as

$$
\int_{0}^{\infty} R_{m}(E) \phi(E) d E=\mu_{\mathrm{m}}, \quad m=1,2, \ldots, M
$$

where $R_{m}(E)$ is the response function, $\phi(E)$ is neutron spectrum, and $\mu_{\mathrm{m}}$ is measurement of the $m^{\text {th }}$ response. Since continuous functions cannot be treated, Eq. (1) is approximated by energy discretization as follows

\footnotetext{
*Corresponding author. Email: jkkim1@hanyang.ac.kr
} 


$$
\sum_{i=1}^{N} R_{m, i} \phi_{i} \approx \mu_{\mathrm{m}}, \quad m=1,2, \ldots, M
$$

where $R_{m, i}$ is the averaged response function, $\phi_{i}$ is the flux in $i^{\text {th }}$ energy bin and $N$ is number of energy bins. These equations can be expressed in matrix form as shown below.

$$
\boldsymbol{R} \vec{\phi}=\vec{\mu}
$$

where, the measurement $\vec{\mu}$ vector and the response matrix $\boldsymbol{R}$ are known values, and the neutron spectrum $\vec{\phi}$ be calculated based on this values. In most cases, there is no inverse of the response matrix because the number of energy bins of the spectrum is greater than the number of response. In other words, $\vec{\phi}$ satisfying the Eq. (3) is not unique.

\subsection{Multi objective genetic algorithm}

Genetic algorithm is an optimization technique that was inspired by natural selection. Natural selection means that some properties increase the chance of survival and reproducing of an individual, thereby producing more offspring. These properties are inherited from the parent to the offspring and become a dominant characteristic in the population during the generation. John Holland developed genetic algorithms, inspired by natural selection. This algorithm has been applied to various problems because it can effectively find the global optimal solution in a wide search space and can be easily applied to nonlinear problems.

\subsubsection{Encoding scheme}

Expressing the solution as sequence of information similar to the genetic form is called encoding, which is essential for implementing a genetic algorithm. There is no clear rule for encoding the solution, and various genetic representations are possible depending on the problem. In this study, the neutron spectrum was expressed using the encoding scheme proposed by Richard M. Vega [9]. They defined several points on the energy axis of the logarithmic scale and then fitted them with the polynomial. Then the polynomial named shifting function was multiplied by the initial guess spectrum to represent the solution.

$$
\begin{array}{r}
\phi_{\text {unfolded }}\left(E_{i}\right)=\phi_{\text {guess }}\left(E_{i}\right) S\left(E_{i}\right) \\
i=1, \ldots, N
\end{array}
$$

where $\phi_{\text {unfolded }}\left(E_{i}\right)$ is the unfolded spectrum, $\phi_{\text {guess }}\left(E_{i}\right)$ is the initial guess spectrum and $S\left(E_{i}\right)$ is shifting function in the $i^{\text {th }}$ energy bin.

\subsubsection{Genetic operator}

A genetic operator is an operator that evolves a population by simulating evolutionary processes. It consists of selection, crossover, and mutation. The selection operator evaluates individuals based on the fitness function, and high-fidelity individuals become parents and deliver genetic information to the next generation. Crossover is the process of inheriting parent's genetic information to offspring. Crossover operator can give a momentum to the evolutionary process because the offspring born to high-fidelity parents are likely to be a better solution for the previous generation. In this algorithm, a single point crossover was used to exchange information. Figure 1 shows the gene expression and shifting function of parents and offspring obtained by the crossover. The points shown in the figure are the genetic information and are defined as the real number in the energy axis. These points are fitted to the third order polynomial to derive the shifting function. The line shown in the figure is shifting function of the individual and this function is multiplied by the initial guess to represent the spectrum from the genetic encoding. Mutation encourages genetic diversity to prevent the solution from falling to a local minimum. The mutation operation was implemented to reset the genetic element if the random number is less than the mutation rate.

(a)

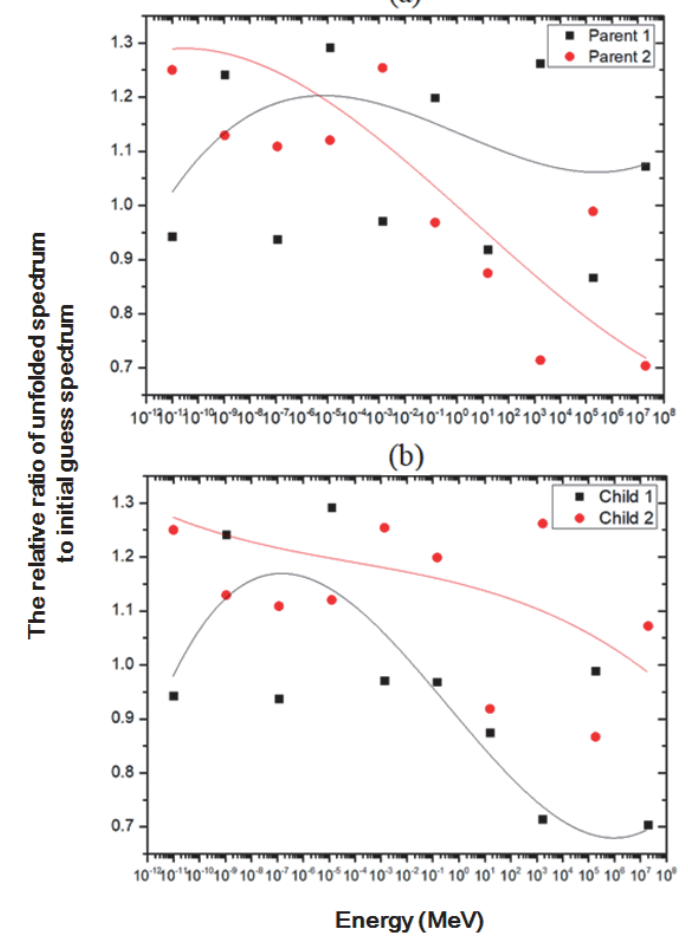

Figure 1. Genotype and polynomial fitting of individuals; (a) Genotype and shift functions of the parents and (b) Genotype and shift functions of the offspring inherited through one-point crossover.

\subsubsection{Fitness functions}

The fitness function determines how individual matches an objective. Solutions with high fitness are selected to inherit their genetic information in the next generation. There are two fitness functions applied in this study. The first goal was to minimize the relative error with the measured values and the second one was 
to maximize the Shannon information entropy, and each term is defined as Eq. (5) and Eq. (6). Shannon proposed a value to quantify information disorder and called it information entropy. It is known that maximizing entropy leads to a probability distribution that best represents the present prior knowledge. This is called the Maximum Entropy Principle.

$$
\begin{aligned}
& \sum_{i=n}^{M} \frac{\left|\left\{\sum_{j=1}^{N} R_{i, j} \phi_{j}\right\}-\mu_{i}\right|}{\mu_{i}} \\
& H=-\sum_{i=n}^{n} p_{i} \log _{2} p_{i}
\end{aligned}
$$

\subsubsection{Pareto front}

Pareto efficiency is a state of solution when it is impossible to create a beneficial change without damaging another objective. The set of Pareto efficient is called the Pareto front. Figure $\mathbf{2}$ shows an optimization problem that minimizes both objectives simultaneously. In this case, each of the solutions has two objective values. Since the solution cannot exist outside the boundary indicated by the red line, the specific objective cannot be decreased without increasing the other objective at the boundary. The solution set of this red line is called Pareto front in this figure.

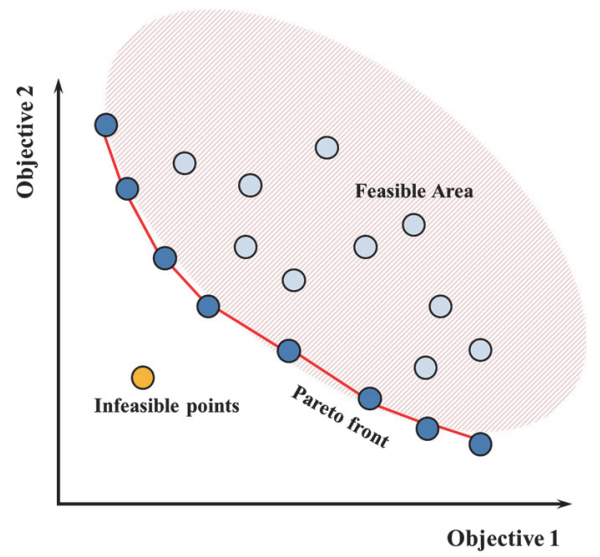

Figure 2. Pareto front of problem minimizing two objectives.

\section{Application}

Numerical problems have been made for applying the algorithm. A parallel neutron beam entered the water and foils were activated by moderated neutrons. The neutron energy was made to follow the Watt fission spectrum. The transport calculation was performed using MCNPX 2.7 code [10], which is highly recommended for neutron and the reaction cross section in ENDF/B -VII library was used.

Four reaction rates for non-threshold reactions and three threshold reactions were calculated. The types and reaction rates of these reactions were listed in Table 1. The upper bound of each energy bin was divided into 100 sections from $10^{-11}$ to $20 \mathrm{MeV}$ and cross sections in each bin were averaged using flat flux weighting. Figure 3 shows the group cross section used in the calculation.

Table 1. Type of reactions used in the problem.

\begin{tabular}{ccc}
\hline Reaction Type & $\begin{array}{c}\text { Threshold } \\
(\mathrm{MeV})\end{array}$ & $\begin{array}{c}\text { Reaction Rate } \\
\left(\mathrm{s}^{-1}\right)\end{array}$ \\
\hline${ }^{27} \mathrm{Al}(\mathrm{n}, \gamma)$ & - & $6.04 \mathrm{E}+11$ \\
${ }^{59} \mathrm{Co}(\mathrm{n}, \gamma)$ & - & $1.09 \mathrm{E}+09$ \\
${ }^{63} \mathrm{Cu}(\mathrm{n}, \gamma)$ & - & $1.57 \mathrm{E}+08$ \\
${ }^{63} \mathrm{Cu}(\mathrm{n}, \alpha)$ & 1.7422 & $1.80 \mathrm{E}+11$ \\
${ }^{197} \mathrm{Au}(\mathrm{n}, \gamma)$ & - & $1.95 \mathrm{E}+06$ \\
${ }^{93} \mathrm{Nb}(\mathrm{n}, 2 \mathrm{n})$ & 8.9600 & $2.14 \mathrm{E}+10$ \\
${ }^{53} \mathrm{Ni}(\mathrm{n}, \mathrm{p})$ & 0.5005 & $7.12 \mathrm{E}+05$ \\
\hline
\end{tabular}

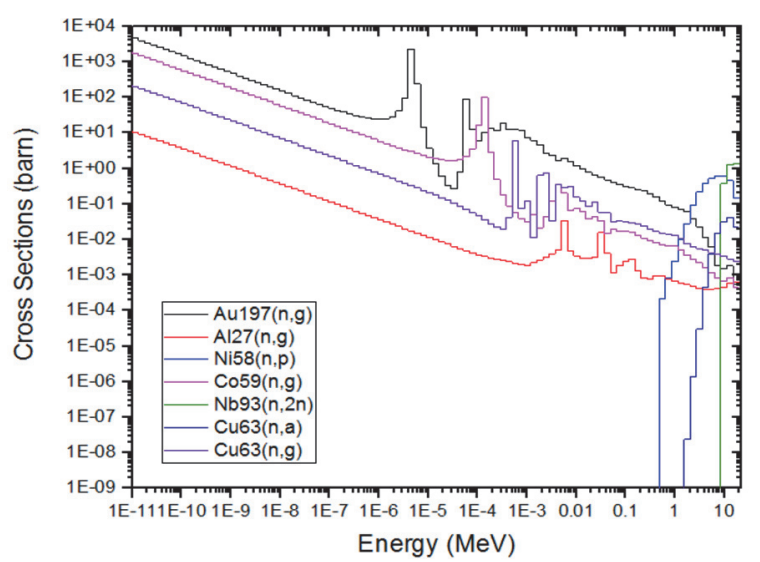

Figure 3. Group reaction cross sections used in the problem.

As described in the previous section, the initial guess spectrum is essential to apply the algorithm. In reality, the initial guess spectrum is inevitably different from the actual value due to various reasons. To ensure that the algorithm works well even in the case of incorrect input, the initial guess was obtained by increasing the water density by $20 \%$ in the problem. It was used as a trial function of the algorithm. As a result of the increased density of water, the initial spectrum was estimated to be high in the low energy region.

In this calculation, the solution is represented by a sequence of 10 real numbers that follow a uniform distribution from 0.6 to 1.4 at initial population. These values were equally spaced on the logarithmic energy axis and fitted with a third order polynomial. The size of population was set to 500. Pareto front was derived using the MATLAB built-in function.

Figure 4 shows Pareto front derived from the genetic algorithm. It is reasonable that solutions located on the Pareto front are more valid than those that are not. Therefore, the neutron spectrum evaluator can drastically reduce the searching space to Pareto front by using multi objective optimization scheme.

Figure 5 shows initial guess and three solutions derived by the algorithm. The position on the Pareto front and objective values are shown in Figure 4. In this problem, solutions located on the Pareto front have almost similar information entropy, so the entropy does not have a significant effect on the results. And the 
lower the relative error of the solution, the more similar to the true spectrum. The solution was stable regardless of the number of iterations.

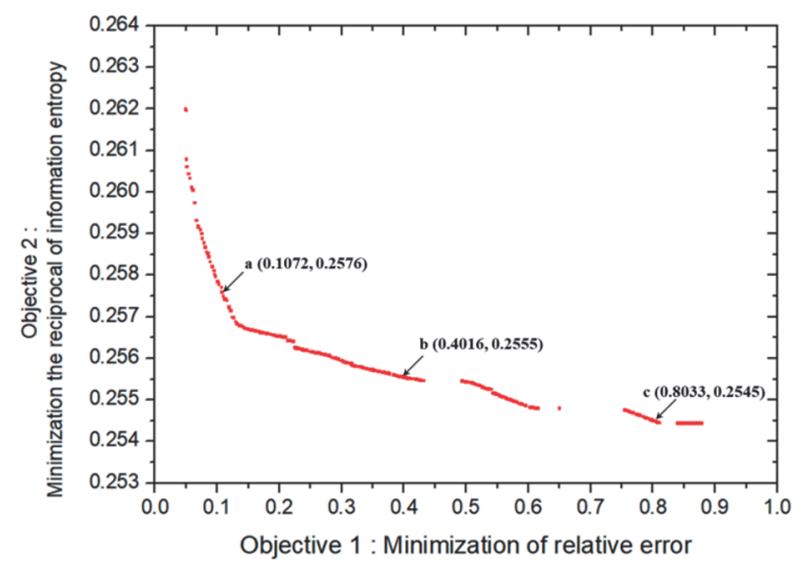

Figure 4. Pareto front derived by genetic algorithm after 300 generations.

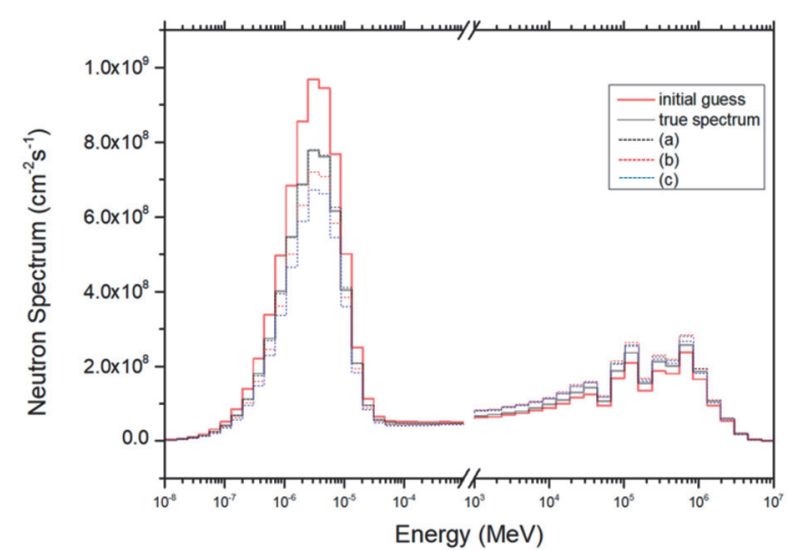

Figure 5. Initial guess and unfolded spectrum reconstructed by genetic multi objective optimization scheme.

\section{Conclusion}

In this study, the neutron spectrum was reconstructed by applying the genetic multi objective optimization scheme. The two objectives of maximizing the Shannon information entropy of the neutron spectrum and minimizing the relative error of the reaction rates were considered. The searching space of the neutron spectrum was limited to Pareto front by genetic algorithm, which allowed us to visually determine the sensitivity of the objectives. Therefore, this approach is expected to strengthen the validity of unfolded spectrum.

\section{Acknowledgements}

This work was supported by the Energy Efficiency \& Resources of the Korea Institute of Energy Technology Evaluation and Planning (KETEP) grant funded by the
Korea government Ministry of Knowledge Economy (2017510300470).

\section{References}

[1] D.J Thomas and A.V Alevra, Bonner sphere spectrometers - a critical review, Nuclear Instruments and Methods in Physics Research Section A: Accelerators, Spectrometers, Detectors and Associated Equipment 476 (2002), pp. 12-20.

[2] S.P. Tripathy, C. Sunil, M. Nandy, P.K. Sarkar, D.N. Sharma and B. Mukherjee, Activation foils unfolding for neutron spectrometry: Comparison of different deconvolution methods, Nuclear Instruments and Methods in Physics Research Section A: Accelerators, Spectrometers, Detectors and Associated Equipment 583 (2007), pp. 421-425.

[3] A. Tarantola, Inverse problem theory and methods for model parameter estimation, Society for Industrial and Applied Mathematics, (2005), pp. 1-40, ISBN 9780898715729.

[4] A. Hoecker and V. Kartvelishvili, SVD approach to data unfolding, Nuclear Instruments and Methods in Physics Research Section A: Accelerators, Spectrometers, Detectors and Associated Equipment 372 (1996), pp. 469-481.

[5] S. Maeda, H. Tomita, J. Kawarabayashi and T. Iguchi, Fundamental study on neutron spectrum unfolding using maximum entropy and maximum likelihood method, Progress in Nuclear Science and Technology 1 (2011). pp. 233-236.

[6] F.Z. Dehimi, A. Seghour and S.E.H. Abaidia, Unfolding of neutron energy spectra with fisher regularisation, IEEE Transactions on Nuclear Science 57 (2010), pp. 768-774.

[7] M. Reginatto, P. Goldhagen and S. Neumann, Spectrum unfolding, sensitivity analysis and propagation of uncertainties with the maximum entropy deconvolution code MAXED, Nuclear Instruments and Methods in Physics Research Section A: Accelerators, Spectrometers, Detectors and Associated Equipment 476 (2002), pp. 242-246.

[8] K. Weise, Mathematical foundation of an analytical approach to Bayesian-statistical Monte Carlo spectrum unfolding, PTB-N-24, Conseil Européen pour la Recherche Nucléaire, (1995).

[9] R.M. Vega and E.J. ParmaVega, Development of a Genetic Algorithm for Neutron Energy Spectrum Adjustment, No. SAND2015-0298C, Sandia National Laboratories (SNL-NM), (2015).

[10]D.B. Pelowitz, MCNPX TM User's Manual Version 2.7.0, LA-CP-11-00438, Los Alamos National Laboratory, (2011). 